\title{
Comparación temporal de la riqueza y composición de insectos inductores de agallas en el dosel de un bosque tropical
}

\author{
Temporal comparison in richness and composition of gall-inducing insects in the rainforest \\ canopy
}

\author{
Enrique Medianero $^{1 *}$, Miguel Paniagua C. ${ }^{1,2}$ y Gabriela Castaño-Meneses ${ }^{3}$ \\ ${ }^{1}$ Programa Centroamericano de Maestría en Entomología, Vicerrectoría de Investigación y Postgrado, Universidad de Panamá \\ ${ }^{2}$ Ingenio Chaparrastique. Carretera al Cuco Km. 144 1/2, San Miguel, El Salvador, C.A. \\ ${ }^{3}$ Ecología y Sistemática de Microartrópodos, Departamento de Ecología y Recursos Naturales, Facultad de Ciencias, Universidad Nacional \\ Autónoma de México. Ciudad Universitaria, 04510 México, D. F \\ *Correspondencia: emedianero@ancon.up.ac.pa; medianero@yahoo.com
}

\begin{abstract}
Resumen. Se compara, entre 2 periodos de muestreos con 9 años de distancia entre sí, la riqueza y composición de especies que forman la comunidad de insectos inductores de agallas en el dosel del Parque Natural Metropolitano (PNM), un bosque seco tropical en la costa del Pacífico de Panamá. Los datos corresponden a muestreos realizados de enero de 1997 a marzo de 1998, y de agosto de 2005 a junio de 2006. Para tener acceso al dosel del bosque se utilizó una grúa instalada en el sitio por el Instituto Smithsonian de Investigaciones Tropicales. Los resultados indican que no existen diferencias significativas en el número de especies de insectos inductores de agallas (29 y 30 respectivamente), en los 2 periodos de muestreo; sin embargo, la comunidad de hace 9 años presenta un reemplazo del $16 \%$ de las especies y se asemeja a la actual en 55\%. Variaciones ambientales y actividades antropogénicas favorecieron la proliferación de lianas y de cecidógenos relacionados con esas plantas. En los 2 periodos de muestreo, tanto árboles como lianas, albergaron un número semejante de insectos inductores de agallas.
\end{abstract}

Palabras claves: árboles, cecidógeno, lianas, Panamá.

\begin{abstract}
A comparison (richness and composition) of the galls inducing insects at canopy forest, in the Parque Natural Metropolitano (PNM), a tropical dry forest of the Pacific coast of Panama was done, in a difference period of 9 years. Sampling was carried out from January 1997 to March 1998 and from August 2005 to June 2006. The crane from the Smithsonian Institution for Tropical Research (STRI) was used in order to access to the canopy. After the results, there are not significant differences in the number of insects which induce the production of galls (29 and 30 respectively), nevertheless the community from 9 years before, presents the replacement of $16 \%$ of the species and is similar in 55\% to the present fauna. Environmental changes and anthropogenic activities have produced the proliferation of vines and cecidogens. In both periods of time, the trees as well as the vines have a similar number galls inducing.
\end{abstract}

Key words: trees, cecidogen, vines, Panama.

\section{Introducción}

En los últimos 20 años, numerosos estudios que documentan la diversidad de insectos inductores de agallas (cecidógenos), y los factores que afectan su riqueza local y global, han sido desarrollados alrededor del mundo (Veldtman y McGeoch, 2003; Raman et al., 2005; NievesAldrey et al., 2008). Sin embargo, pocas investigaciones han incluido un componente temporal en la estimación de la riqueza de los artrópodos con este hábito (Medianero y Barrios, 2001; Maia y Fernandes, 2004; Dalbem y

Recibido: 21 abril 2009; aceptado: 12 octubre 2009
Mendonça, 2006; Nieves-Aldrey et al., 2008), y ninguno ha abordado las variaciones en la riqueza de especies de tales comunidades a largo plazo.

La diversidad muestra variaciones considerables que conforman un proceso natural en los ecosistemas, y han ocurrido a lo largo de la historia biológica (Ulfstrand, 1992). Sin embargo, existen fenómenos que promueven, aceleran y potencian dichas modificaciones. Actividades antropogénicas como la sobreexplotación de hábitat, la deforestación, la contaminación, y la introducción de especies exóticas, entre otras, están consideradas como las principales fuerzas que conducen los cambios en la diversidad y composición de las comunidades alrededor 
del mundo (Spooner, 2005; McKinney, 2006; Roe et al., 2006; Coates et al., 2006). En los últimos años, ha crecido el interés por cuantificar el efecto de tales actividades en las comunidades de artrópodos (Basset et al., 1998), sin embargo, son pocos los datos que se tienen de sus efectos en las comunidades de artrópodos del dosel del bosque tropical (Davis y Sutton, 1998). Dado que el dosel de los bosques constituye la interfase funcional entre más del 90\% de la biomasa terrestre y la atmósfera (Ozanne et al., 2003), es uno de los ambientes clave en el mantenimiento de la biodiversidad global, ya que en él se realizan diversos procesos esenciales de los ecosistemas (Barker, 1996). Es también el estrato del bosque donde se ha registrado la mayor diversidad de algunos grupos de insectos, incluyendo los inductores de agallas y minadores de hojas (Medianero et al., 2003; Ribeiro y Basset, 2007).

Los insectos inductores de agallas son un grupo con gran diversidad; el número promedio de las estimaciones realizadas sobre la riqueza, sugiere un valor de 132930 especies (Espírito-Santo y Fernandes, 2007). Aunado a ello, poseen características que los hacen modelos muy interesantes para estudios ecológicos. Debido a su inmovilidad como cecidia (agalla), son fáciles de detectar; además, son fácilmente reconocibles, puesto que las cecidias son la expresión específica de la biología del insecto y su morfología y complejidad, el fenotipo extendido de las especies inductoras (Stone y Cook, 1998). Presentan además una alta especificidad, infestando generalmente una sola especie de planta o especies de un mismo género (Dreger-Jauffret y Shorthouse, 1992), por lo que cualquier modificación en el medio que produzca cambios en la composición vegetal, se reflejará en la riqueza y composición de las comunidades de los inductores de agallas.

El presente estudio pretende determinar si al cabo de 9 años han ocurrido cambios de riqueza y composición en la comunidad de insectos inductores de agallas que habitan el dosel de un bosque seco tropical en la costa Pacífica de Panamá. Debido a variaciones ocurridas en las condiciones ambientales y a las actividades antropogénicas durante la última década, en particular la construcción de carreteras cerca del sitio de estudio (Heckadon-Moreno, 2005), se espera que exista una disminución en la riqueza, así como variación en la composición de las especies que forman la comunidad de insectos inductores de agallas.

\section{Materiales y métodos}

En el estudio se comparan datos obtenidos de enero de 1997 a marzo de 1998 (15 meses de muestreo) y de agosto de 2005 a junio de 2006 (11 meses de muestreo).
Los muestreos fueron realizados en el dosel del bosque del Parque Natural Metropolitano (PNM), ubicado en la Provincia de Panamá ( $8^{\circ} 59^{\prime} \mathrm{N}$ y $79^{\circ} 33^{\prime} \mathrm{O}$ ). Para tener acceso al dosel se utilizó una grúa instalada por el Instituto Smithsonian de Investigaciones Tropicales (STRI) que cubre aproximadamente 0.85 ha de bosque (Fig. 1). La altitud en el sitio de la grúa es de $30 \mathrm{~m}$ snm, con una temperatura promedio anual de $26.3^{\circ} \mathrm{C}$ y una precipitación anual de $1865 \mathrm{~mm}$. La vegetación corresponde a un bosque seco tropical, caracterizado por la dominancia de árboles deciduos y lianas en el dosel (Basset et al., 2003). La altura del dosel es de $30 \mathrm{~m}$, con árboles que emergen hasta los $40 \mathrm{~m}$. Se han identificado 40 especies de árboles y 35 de lianas en el área que cubre el radio de la grúa (Ødegaard, 2000).

Para determinar la riqueza de especies de insectos inductores de agallas en la comunidad de plantas del dosel del bosque en 1997, se marcaron 53 especies (80 individuos) entre árboles y lianas, y se revisó un área foliar aproximada de $93.521 \mathrm{~m}^{2}$ (Medianero et al., 2003). El marcado y los muestreos se realizaron en transectos circulares, desplazando el brazo de la grúa en sentido de las manecillas del reloj. Las plantas fueron elegidas al azar, partiendo de un punto y marcándolas con cinta tal y como se fueron encontrando. De cada especie de planta se marcó más de 1 individuo; de los árboles maduros, de los que sólo había un ejemplar en el área de la grúa, se marcaron 3 ramas del mismo individuo. Todos los individuos fueron codificados y muestreados cada 2 semanas durante cada periodo de estudio. Una vez que se llegaba a una planta se procedía a registrar por aproximadamente 5 minutos la presencia de agallas en los diferentes órganos. En 20052006 se hizo la revisión semanal de las especies de plantas que habían sido marcadas (una semana los árboles y la otra las lianas); hubo especies que 9 años después ya no se

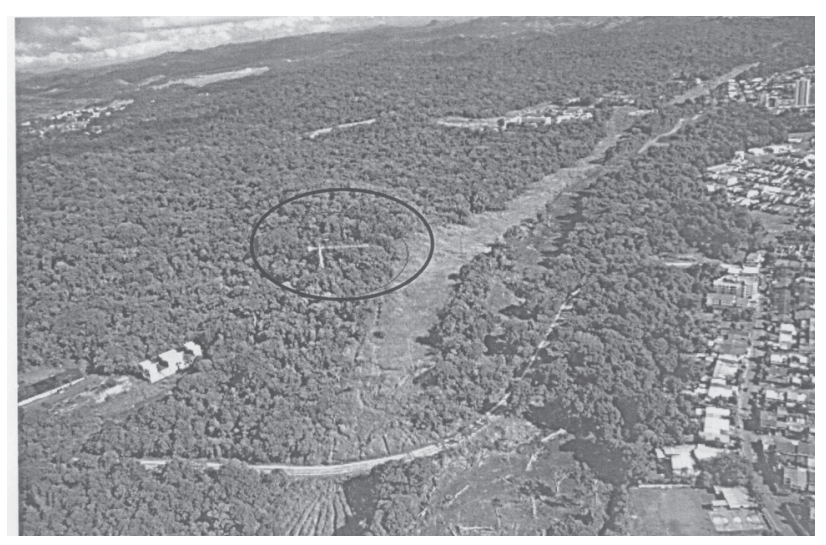

Figura 1. Vista aérea del sitio de la grúa instalada en el Parque Natural Metropolitano, costa del Pacífico, Panamá. 
encontraron en el área de la grúa. Durante los 2 periodos de muestreo, los órganos de la planta donde se encontraron agallas (hojas, tallos, frutos, yemas y raíces) fueron colocados en bolsas plásticas, trasladados al laboratorio y revisados cada 2 días, por un periodo mínimo de 1 mes, siguiendo la metodología propuesta por Gagné (1994).

En el laboratorio, las agallas fueron fotografiadas y, dependiendo del número colectado, el 50\% se diseccionó para la identificación de la larva al nivel de familia. Los adultos emergidos fueron colocados en alcohol al $70 \%$ y permanecen en la colección del Programa Centroamericano de Maestría en Entomología de la Universidad de Panamá. Los morfotipos de insectos inductores de agalla se separaron de acuerdo a la morfología externa de la agalla, la larva, la planta hospedera y el adulto (en los casos en que se obtuvo). En este tipo de estudio, cada morfotipo de agalla se considera único para cada especie de inductor (Fernandes y Price, 1988; Price et al., 1998).

Los datos de las variables climáticas, registradas diariamente en el PNM desde 1995 por una estación meteorológica instalada en la torre de la grúa, indican que a lo largo del tiempo se han presentado variaciones, las cuales incluyen disminución en la precipitación y en la humedad relativa del aire; por el contrario, la radiación solar se ha incrementado a través de los años (Fig. 2). La temperatura promedio no muestra un cambio definido a lo largo del tiempo. Adicionalmente, en la última década, se construyó un corredor vehicular de 6 carriles (Fig. 1) próximo al sitio donde se encuentra la grúa $(200 \mathrm{~m})$, lo que convirtió este lugar en el área de amortiguación del corredor vehicular.
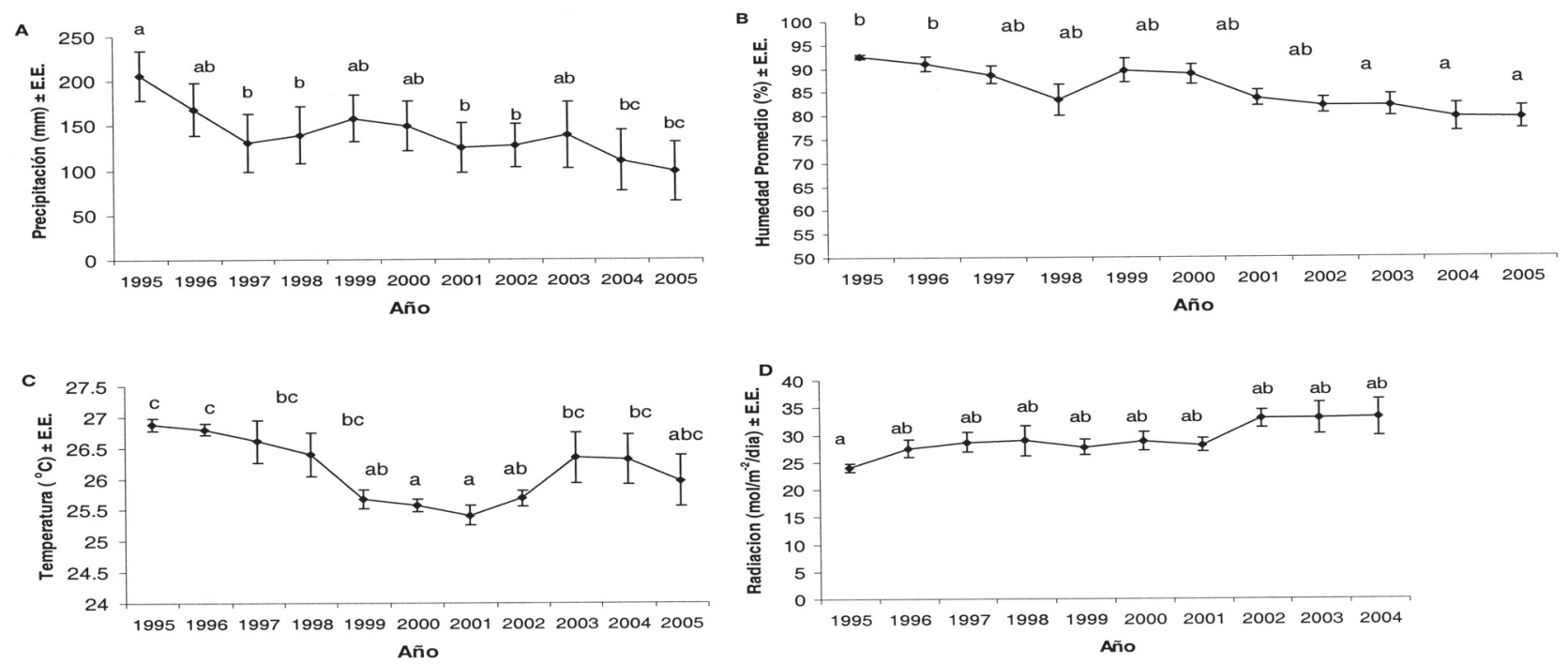

Figura 2. Variación promedio anual (con desviaciones estándar) de las principales variables ambientales registradas de 1995 a 2005 en el Parque Natural Metropolitano, costa del Pacífico, Panamá.
Para verificar la eficiencia de los muestreos se construyeron curvas de rarefacción con BioDiversity Professional Ver. 2 (McAleece, 1997). A los datos de número de morfotipos de agallas encontrados en los 2 periodos se les aplicó una prueba de bondad de ajuste con $J i$ cuadrada $\left[(\mathrm{O}-\mathrm{E})^{2} / \mathrm{E}\right]$. Para determinar cambios en el número de agallas por hábito de crecimiento de las plantas hospederas (árboles y lianas), se utilizó la prueba de MacNemar. Los análisis se realizaron con el programa Statistica ver. 6.0 (Statsoft, 1995). Para determinar cambios en la composición de la comunidad de especies inductoras de agallas entre 1997-98 y 2005-06, se calcularon 2 medidas de diversidad $\beta$, siguiendo a Koleff (2005), la primera medida fue el coeficiente de similitud de Jaccard $(\beta \mathrm{j})$, que implícitamente incorpora diferencias en la composición atribuibles a gradientes de diversidad, pero ignora la magnitud relativa de las especies ganadas y las especies perdidas. La segunda medida fue el coeficiente de Routledge $(\beta \mathrm{r})$, que se enfoca más en las diferencias en composición, que en las diferencias en riqueza de especies. El $\beta_{\mathrm{r}}$ presenta una variación entre 0 a 100 y es una modificación del índice de Shannon (Routledge, 1977).

$$
\beta_{\mathrm{r}}=\mathrm{S}^{2} / 2 \mathrm{r}+\mathrm{S}
$$

Donde: $\mathrm{S}=$ número total de especies registradas en ambos muestreos.

$\mathrm{r}=$ número pares de especies cuya distribución se sobrepone. 
Cuadro 1. Especies de plantas hospederas de insectos cecidógenos en el dosel del PNM durante los muestreos realizados en 1997-1998 y 2005-2006. L (lianas) = 15 especies, A (árbol) $=13$ especies, + = presencia, - = ausencia

\begin{tabular}{|c|c|c|c|c|c|}
\hline $\begin{array}{l}\text { Morfoespecie inductor } \\
\text { de cecidia }\end{array}$ & Familia & Especie & $\begin{array}{c}\text { Hábito } \\
\text { del hospedero }\end{array}$ & $\begin{array}{c}\text { Primer } \\
\text { muestreo }\end{array}$ & $\begin{array}{l}\text { Segundo } \\
\text { muestreo }\end{array}$ \\
\hline Cecidomyiidae sp. 1 & Fabaceae & Enterolobium cyclocarpum & A & + & + \\
\hline Cecidomyiidae sp. 2 & Fabaceae & Enterolobium cyclocarpum & A & + & + \\
\hline Cecidomyiidae sp. 3 & Fabaceae & Enterolobium cyclocarpum & A & + & + \\
\hline Cecidomyiidae sp. 4 & Boraginaceae & Cordia alliadora & A & + & + \\
\hline Cecidomyiidae sp. 5 & Boraginaceae & Cordia alliadora & A & + & + \\
\hline Cecidomyiidae sp. 6 & Lauraceae & Nectandra cuspidata & $\mathrm{A}$ & + & - \\
\hline Cecidomyiidae sp. 7 & Bignoniaceae & Amphilophium paniculatum & $\mathrm{L}$ & + & - \\
\hline Cecidomyiidae sp. 8 & Bignoniaceae & Amphilophium paniculatum & $\mathrm{L}$ & + & - \\
\hline Cecidomyiidae sp. 9 & Asteraceae & Mikania leiostachya & $\mathrm{L}$ & + & + \\
\hline Cecidomyiidae sp. 10 & Asteraceae & Mikania leiostachya & $\mathrm{L}$ & + & + \\
\hline Cecidomyiidae sp. 11 & Moraceae & Castilla elastica & A & + & + \\
\hline Cecidomyiidae sp. 12 & Dilleniaceae & Doliocarpus major & $\mathrm{L}$ & + & + \\
\hline Cecidomyiidae sp. 13 & Sapindaceae & Serjania mexicana & $\mathrm{L}$ & + & + \\
\hline Cecidomyiidae sp. 14 & Vitaceae & Vitis tiliifolia & $\mathrm{L}$ & + & + \\
\hline Cecidomyiidae sp 15 & Vitaceae & Vitis tiliifolia & $\mathrm{L}$ & + & + \\
\hline Cecidomyiidae sp. 16 & Sapotaceae & Chrysophyllum cainito & A & + & - \\
\hline Cecidomyiidae sp. 17 & Combretaceae & Combretum decandrum & $\mathrm{L}$ & + & - \\
\hline Cecidomyiidae sp. 17 & Combretaceae & Combretum fruticosum & $\mathrm{L}$ & + & + \\
\hline Cecidomyiidae sp. 18 & Combretaceae & Combretum fruticosum & $\mathrm{L}$ & + & + \\
\hline Cecidomyiidae sp. 19 & Sapindaceae & Cupania latifolia & A & + & - \\
\hline Cecidomyiidae sp. 20 & Moraceae & Ficus insipida & $\mathrm{A}$ & + & + \\
\hline Cecidomyiidae sp. 21 & Moraceae & Cecropia longipes & A & + & + \\
\hline Cecidomyiidae sp. 21 & Moraceae & Cecropia obtusifolia & A & + & + \\
\hline Cecidomyiidae sp. 21 & Moraceae & Cecropia peltata & $\mathrm{A}$ & + & - \\
\hline Cecidomyiidae sp. 22 & Bignoniaceae & Phryganocydia corymbosa & $\mathrm{L}$ & + & + \\
\hline Cecidomyiidae sp. 23 & Moraceae & Cecropia obtusifolia & $\mathrm{A}$ & + & + \\
\hline Cecidomyiidae sp. 24 & Tiliaceae & Luehea speciosa & A & + & + \\
\hline Cecidomyiidae sp. 25 & Rhamnaceae & Gouania lupuloides & $\mathrm{L}$ & + & - \\
\hline Cecidomyiidae sp. 26 & Loranthaceae & Psittcanthus calyculatus & $\mathrm{L}$ & + & - \\
\hline Cecidomyiidae sp. 27 & Anacardiaceae & Spondias mombin & $\mathrm{A}$ & - & + \\
\hline Cecidomyiidae sp. 28 & Anacardiaceae & Spondias mombin & A & - & + \\
\hline Cecidomyiidae sp. 29 & Phytolaccaceae & Trichostigma octandrum & $\mathrm{L}$ & - & + \\
\hline Cecidomyiidae sp. 30 & Bignoniaceae & Arrabidea patellifera & $\mathrm{L}$ & - & + \\
\hline Cecidomyiidae sp. 31 & Vitaceae & Vitis tiliifolia & $\mathrm{L}$ & - & + \\
\hline Cecidomyiidae sp. 32 & Bignoniaceae & Paragonia pyramidata & $\mathrm{L}$ & - & + \\
\hline Cecidomyiidae sp. 33 & Fabaceae & Machaerium riparium & $\mathrm{L}$ & - & + \\
\hline
\end{tabular}




\begin{tabular}{|c|c|c|c|c|c|}
\hline $\begin{array}{l}\text { Morfoespecie inductor } \\
\text { de cecidia }\end{array}$ & Familia & Especie & $\begin{array}{c}\text { Hábito } \\
\text { del hospedero }\end{array}$ & $\begin{array}{l}\text { Primer } \\
\text { muestreo }\end{array}$ & $\begin{array}{l}\text { Segundo } \\
\text { muestreo }\end{array}$ \\
\hline Cecidomyiidae sp. 34 & Bignoniaceae & Cydista sp. & $\mathrm{L}$ & - & + \\
\hline Cecidomyiidae sp. 35 & Sapotaceae & Chrysophyllum cainito & A & - & + \\
\hline H. thoracica (Buprestidae) & Bignoniaceae & Amphilophium paniculatum & $\mathrm{L}$ & + & + \\
\hline Cerambycidae sp. 1 & Lauraceae & Cinnamomun triplinerve & A & + & + \\
\hline
\end{tabular}

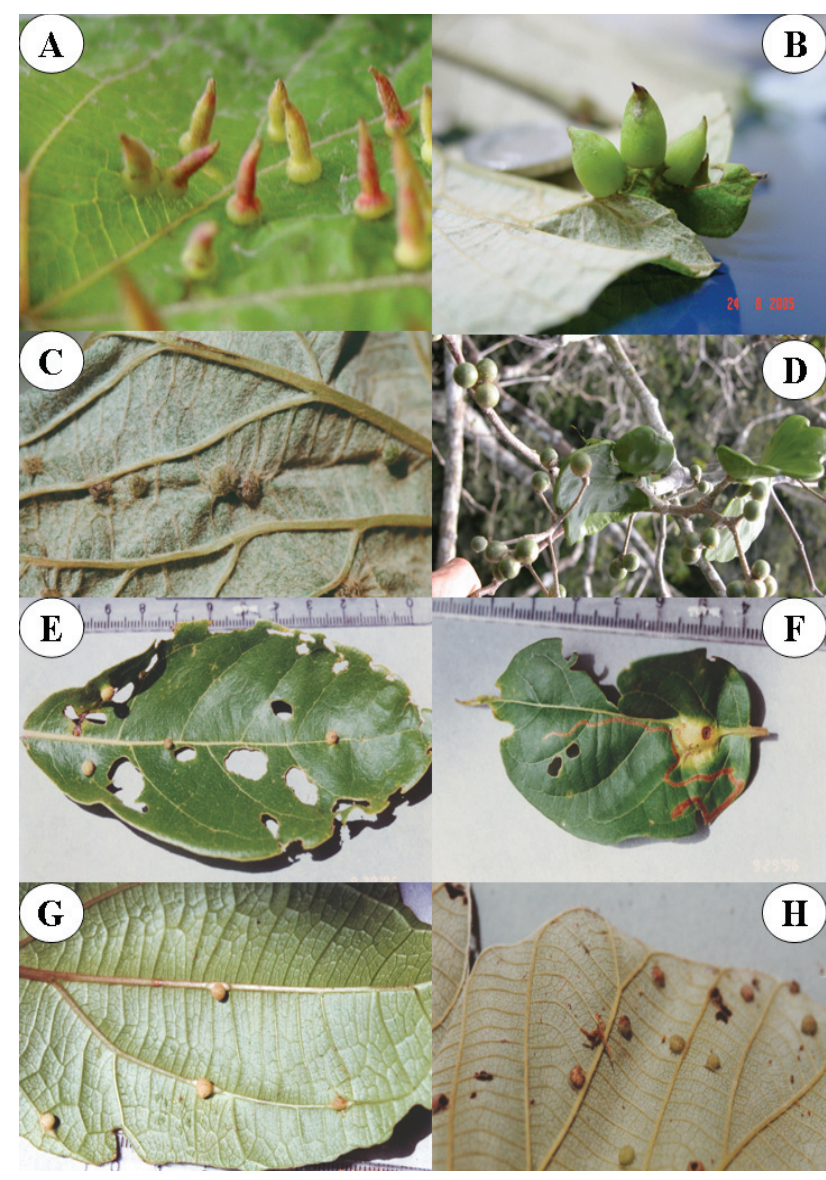

Figura 3. Cecidias inducidas por especies de la familia Cecidomyiidae sobre: (A-C) Vitis tiliifolia; (D) Enterolobium cyclocarpum; (E) Cordia alliadora; (F) Combretum fruticosum; (G) Mikania leiostachya; (H) Cecropia longipes.

\section{Resultados}

Durante los 2 periodos de muestreo se colectaron en total 37 morfotipos de agallas inducidas por insectos sobre 28 especies de plantas, las cuales se incluyen en 25 géneros y 16 familias (Cuadro 1). Tales resultados representan el
$52 \%$ de las plantas marcadas y el $37 \%$ de las plantas de la comunidad del dosel del PNM. El 95\% de las cecidias fueron inducidas por especies de la familia Cecidomyiidae (Diptera) (Fig. 3), el 5\% restante fue inducido por 2 especies del orden Coleoptera: Hylaeogena thoracica (Buprestidae) y una especie de la familia Cerambycidae. El $89.74 \%$ de las cecidias fueron inducidas en hojas, 5.2\% en tallos y $2.5 \%$ en botones florales y en raíces de epífitas. Fueron infestadas 3 especies de plantas (Amphilophium paniculatum, Enterolobium cyclocarpum y Vitis tiliifolia) por 3 especies de inductores; 5 especies por 2 inductores y las restantes sirvieron de hospedero a 1 sola especie de inductor de agalla; 2 especies de la familia Cecidomyiidae tuvieron más de 1 especie de planta hospedadora, pero del mismo género (Cuadro 1).

Las curvas de rarefacción para ambos periodos de muestreo alcanzaron la asíntota, lo que indica que se alcanzó el número máximo de morfotipos presentes en el área (Fig. 4). En el primer periodo de muestreo (199798) se colectaron 29 morfotipos de agallas, y 30 en el segundo periodo (2005-06). No se encontraron diferencias significativas en el número de morfotipos de agallas en los 2 periodos de muestreo $\left(\chi^{2}=0.07, p>0.05, g l=1\right)$.

De los 37 morfotipos de agallas encontradas, 17 estuvieron asociadas a árboles y 20 a lianas. En el primer periodo de muestreo el número de especies de árboles que sirvieron de hospedadores fue mayor en comparación con las lianas, pero en el segundo, esta relación se invirtió (Cuadro 2); sin embargo, no se encontraron diferencias significativas en el número de inductores de agallas asociados a árboles o lianas en ninguno de los 2 periodos $\left(\chi^{2}=0.83 \mathrm{p}>0.05\right)$.

Del total de cecidias encontradas, 21 fueron compartidas en los 2 muestreos, 8 fueron únicas en el primero y 9 para el segundo. De los 8 morfotipos encontrados sólo en el primer muestreo, 3 se asociaron con árboles y 5 con lianas. De los encontrados sólo en el segundo muestreo, 3 estuvieron asociados a árboles y 6 con lianas. El coeficiente de similitud de Jaccard $\left(\beta_{\mathrm{j}}\right)$ indica una semejanza de especies entre los periodos de $55 \%$ mientras que el coeficiente de Routledge $\left(\beta_{\mathrm{r}}\right)$ indica que ha ocurrido un reemplazo del $16.33 \%$ de las especies del primer periodo de muestreo. 


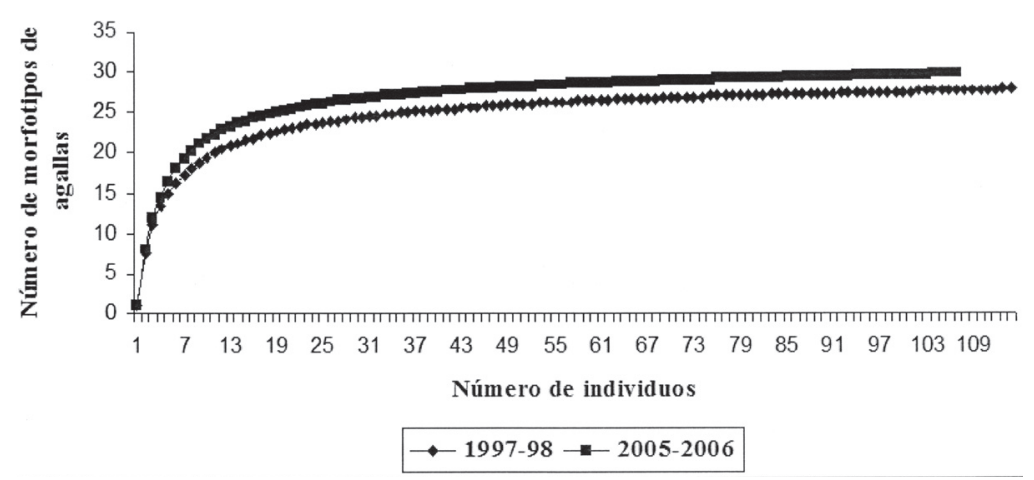

Figura 4. Curvas de rarefacción para los 2 periodos de muestreos. de la dinámica de las comunidades del dosel en los bosques tropicales (Basset et al., 1998), nos limita en determinar si el tiempo trascurrido entre el primero y segundo muestreo es el necesario para que las comunidades respondan de una manera más rápida a los disturbios y variaciones ambientales registradas. De cualquier manera, como consecuencia a su condición de monofagos la presencia de las especies de cecidógenos en una determinada comunidad estará determinada por la presencia, abundancia, condición fisiológica y fenológica de su planta hospedera (Weis et al., 1988).

Cuadro 2. Número de cecidias encontradas y la relación con el hábito de crecimiento de su planta hospedera durante 2 periodos de muestreo en el dosel del PNM (no se encontraron diferencias estadísticas $\left(\chi^{2}=0.83 \mathrm{p}>0.05\right)$

\begin{tabular}{lcc}
\hline Número de especies & 1997-1998 & 2005-2006 \\
\hline Cecidias & 29 & 30 \\
Árboles hospederos & 12 & 10 \\
Lianas hospederas & 10 & 12 \\
Cecidias en árboles & 14 & 14 \\
Cecidias en lianas & 14 & 15 \\
\hline
\end{tabular}

\section{Discusión}

Si bien es un hecho que los disturbios y las variaciones climáticas están entre los más importantes eventos que provocan heterogeneidad temporal y espacial en la estructura y dinámica natural de las comunidades (Sousa, 1984), también es cierto que las comunidades son determinadas por las interacciones entre sus miembros, y entre éstos y los componentes bióticos y abióticos del ecosistema, por lo que no hay una idea clara de cómo los cambios climáticos y las perturbaciones pueden influir en la riqueza y composición de las comunidades de artrópodos (Gobbi et al., 2006). Los resultados obtenidos en este estudio indican que a pesar de las variaciones en las condiciones ambientales (Fig. 2), y el efecto de borde provocado por la construcción de una autovía cerca del sitio de la grúa, la comunidad de inductores de agallas encontrados en el dosel del bosque no se ha modificado significativamente en los 2 periodos de muestreos, separados por nueve años. Los 9 morfotipos de especies inductoras de agallas encontradas en el segundo muestreo y 8 del primer periodo que no fueron encontradas, indican que aunque el remplazo de especies es bajo (16.33\%) éste está ocurriendo. El poco conocimiento que se tiene
Lo que significa que aunque existan nuevas especies de plantas en el área de estudio, si éstas no son hospederas de algún inductor de agalla, la presencia de esta planta no afectará la composición de la comunidad de agalladores. $\mathrm{Si}$ por el contrario, los cambios benefician la presencia y abundancia de una especie de planta vinculada a inductores de agallas en el área, entonces las poblaciones de estos insectos relacionados a ella se verán beneficiadas. Debido a la dependencia que presenta el inductor por su planta hospedera, los orígenes de esta asociación hay que buscarlos en la historia evolutiva de ambas especies, donde, características principalmente químicas de las plantas, las hacen susceptibles a un determinado insecto que logra controlar el desarrollo de los tejidos de la planta e inducir la formación de la agalla (Nieves-Aldrey, 2001). En nuestros resultados, se refleja este comportamiento, ya que las modificaciones ambientales y antropogénicas en el sitio de estudio parece haber favorecido la presencia de lianas, lo que ha sido benéfico para un grupo de insectos cecidógenos. Los resultados apoyan los obtenidos por Ødegaard (2000), quien sugiere que en el dosel del bosque tropical los insectos fitófagos están asociados en igual número de especies a lianas que a árboles maduros, lo que tiene una importancia en el mantenimiento de la diversidad local.

Con frecuencia, la variación temporal de las especies de una comunidad es menos estudiada que su distribución espacial (Novotny y Weiblen, 2005), por lo que esta investigación aporta datos de la dinámica de un grupo de artrópodos con el hábito de inducir agallas en un estrato del bosque poco explorado. Los resultados obtenidos apoyan la idea que en las comunidades tropicales la composición y estructura de la vegetación es el factor clave que determina la presencia o ausencia de las distintas especies de insectos cecidógenos, y que en el bosque tropical las lianas son un factor fundamental en el mantenimiento de la diversidad local. 


\section{Agradecimientos}

Al Instituto Smithsonian de Investigaciones Tropicales por las facilidades prestadas para el uso de la grúa; a Mirna Samaniego (STRI), por la ayuda con la identificación de especies de plantas y por sus valiosos comentarios al manuscrito, a Héctor Barrios (Universidad de Panamá), por sus atinadas sugerencias al manuscrito. A Marcos Guerra, por la foto de la grúa. EM es financiado por una beca del gobierno de Panamá (IFARHU-SENACYT) para realizar estudios doctorales. MRP fue patrocinado por una beca del Programa Regional del DAAD. La participación de GCM fue gracias a una beca posdoctoral otorgada por la Dirección General de Asuntos del Personal Académico, Universidad Nacional Autónoma de México. Los datos climáticos fueron cortesía del Terrestrial Environmental Science Program (STRI) y Steve Paton.

\section{Literatura citada}

Barker, M. G. 1996. Vertical profiles in a Brunei rain forest: I. Microclimate associated with a canopy tree. Journal of Tropical Forest Science 8:505-519.

Basset, Y., V. Novotny, S. Miller y N. Springates. 1998. Assessing the impact of forest disturbance on tropical invertebrates; some comments. Journal of Applied Ecology 35:461-466.

Basset, Y., V. Horlyck y J. S Wright. 2003. Studying forest canopies from above: The International Canopy Crane Network. Smithsonian Tropical Research Institute and UNEP, Panama. 196 p.

Coates, F., I. D. Lunt y R. L. Tremblay. 2006. Effects of disturbance on population dynamics of the threatened orchid Prasophyllum correctum D.L. Jones and implications for grassland management in the south-eastern Australia. Biological Conservation 129:59-69.

Dalbem, R. V. y M. S. Mendonça. 2006. Diversity of galling arthropods and host plants in a subtropical forest of Porto Alegre, Southern Brazil. Neotropical Entomology 35:616624.

Davis, A. y S. Sutton. 1998. The effects of rainforest canopy loss on arboreal dung beetles in Borneo: implications for the measurement of biodiversity in derived tropical ecosystems. Biodiversity Research 4:167-173.

Dreger- Jauffret, F. y J. D. Shorthouse. 1992. Diversity of gall-inducing insects and their galls. In Biology of InsectInduced Galls, J. D. Shorthouse y O. Rohfritsh (eds.). Oxford University Press, New York. p. 8-33.

Espírito-Santo, M. M. y G. W. Fernandes. 2007. How many species of gall-inducing insects are there on the Earth, and where they are? Annals of Entomological Society of America 100:95-99.

Fernandes, G. W. y P. Price. 1988. Biogeographical gradients in galling species richness: test of hypothesis. Oecologia 76:161-167.

Gagné, R. J. 1994. The gall midges of the neotropical region.
Cornell University Press, Ithaca, New York. 352 p.

Gobbi, M., D. Fontanero y F. De Bernardi. 2006. Influence of climate on animal communities in space and time: case of spider assemblages along an alpine glacier foreland. Global Change Biology 12:1985-1992.

Heckadon-Moreno, S. 2005. Light and shadows in the management of the Panama Canal Watershed. In The Río Chagres, Panama - A multidisciplinary profile of a tropical watershed, R. S. Harmon (ed.). Springer, The Hague. p. 29-44.

Koleff, P. 2005. Conceptos y medidas de la diversidad beta. In Sobre la diversidad: el significado de las diversidades alfa, beta y gama, G. Halffer, J. Soberón, P. Koleff y A. Melic (eds.). Monografías del tercer milenio, vol. 4. GORFI, Zaragoza. p. 19-40.

Maia, V. C. y G. W. Fernandes. 2004. Insect gall from Serra de São José (Tiradentes, MG, Brazil). Brazilian Journal of Biology 64:423-445.

Mani, M. S. 1964. The ecology of plant galls. Dr. W. Junk, The Hague. 434 p.

McAleece, N. 1997. Biodiversity professional. beta. Version 2.0. The Natural History Museum / The Scottish Association for Marine Science.

Mckinney, M. 2006. Urbanization as a major cause of biotic homogenization. Biological Conservation 127:247-260.

Medianero, E. y H. Barrios 2001. Riqueza de insectos cecidógenos en el dosel y sotobosque de dos zonas ecológicas en Panamá. Scientia (Panamá) 16:17-42

Medianero, E., A. Valderrama y H. Barrios. 2003. Diversidad de insectos minadores de hojas y formadores de agallas en el dosel y sotobosque del bosque tropical. Acta Zoológica Mexicana (n.s) 89:153-168

Nieves-Aldrey, J. L. 1998. Insectos que inducen la formación de agallas en las plantas; una fascinante interacción ecológica y evolutiva. Boletín de la Sociedad Entomológica Aragonesa 23:3-12.

Nieves-Aldrey, J. L. 2001. Hymenoptera, Cynipidae. In Fauna ibérica, vol. 16, M. A. Ramos (ed.). Museo Nacional de Ciencias Naturales, CSIC, Madrid. 636 p.

Nieves-Aldrey, J. L., A. Ibañez y E. Medianero. 2008. Richness and composition of gall-inducing arthropods at Coiba National Park, Panama. Revista de Biología Tropical 56:1269-1286.

Novotny, V. y G. D. Weiblen. 2005. From communities to continents: beta diversity of herbivorous insects. Annales Zoologici Fennici 42:463-475.

Ødegaard, F. 2000. The relative importance of trees versus lianas as host for phytophagous beetles (Coleoptera) in tropical forest. Journal of Biogeography 27:283-296.

Ozanne, C. M. P., D. Anhuf, S. L. Boulter, M. Keller, R. L. Kitching, D. Körner, F. C. Meinzer, A. W. Mitchell, T. Nakashuizuka, P. L. Silva Dias, N. E. Stork, S. J. Wright y M. Yoshimura. 2003. Biodiversity meets the atmosphere: a global view of forest canopies. Science 301:183-186.

Price, P., G.W. Fernandes, A. C. F. Lara, J. Brawn, H. Barrios, M. G. Wright, S. Ribeiro y N. Rothcliff. 1998. Global patterns in local number of insects galling species. Journal of Biogeography 25:581-591.

Raman, A., C. W. Schaefer y T. M. Withers. 2005. Galls and gall- 
inducing arthropods: an overview of their biology, ecology and evolution. In Biology, ecology, and evolution of gallinducing arthropods, A. Raman, C. Schaefer y T. Withers (eds.). Science, India. p. 1-33.

Ribeiro, S. P. y Y. Basset. 2007. Gall forming and free-feeding herbivory along vertical gradients in a lowland tropical rainforest: the importance of leaf sclerophylly. Ecography 60:663-672

Roe, J., J. Gibson y B. Kingbury. 2006. Beyod the wetland border: Estimating the impact of roads for two species of water snakes. Biological Conservation 130:161-168.

Routledge, R. D. 1977. On Whittaker's components of diversity. Ecology 58:1120-1127.

Sousa, W. 1984. The role of disturbance in natural communites. Annual Review of Ecology Evolution and Systematics 15:353-391.

Spooner, P. 2005. Response of Acacia species to disturbance by roadwords in roadside environments in southern New Wales, Australia. Biological Conservation 122:231-242.

StatSoft.1995. Statistical user guide. Complete statistical system. Statsoft, Tulsa, Oklahoma,.

Stone, G. H. y J. M. Cook. 1998. The structure of cynipid oak galls: patterns in the evolution of an extended phenotype. Proceedings of the Royal Society of London B. 265:979988.

Ulfstrand, S. 1992. Biodiversity. How to reduce its decline. Oikos 63:3-5.

Veldtman, R. y M. A. McGeoch. 2003. Gall-forming insect species richness along a non-scleromorphic vegetation rainfall gradient in South Africa: the importance of plant community composition. Austral Ecology 28:1-13.

Weis, A. E., R. Walton y C. L. Crego.1988. Reactive plant tissue sites and population biology of gall makers. Annual Review of Entomology 33:467-486. 